\title{
Systemic Inflammation and Seminal Parameters in Chronic
} Hemodialysis Patients

\author{
Gilmar Pereira Silva, ${ }^{1,}$ Fabiana Pirani Carneiro, ${ }^{2}$ and Vitor Pereira Xavier Grangeiro ${ }^{3}$ \\ ${ }^{1}$ Urologist MD of the University of Brasilia, Brasilia, Federal District, Brazil \\ ${ }^{2}$ Professor PhD at the University of Brasilia, Brasilia, Federal District, Brazil \\ ${ }^{3}$ Academic of the Faculty of Medical Sciences, Joao Pessoa, Paraiba, Brazil \\ "Corresponding author: Gilmar Pereira Silva, Hotel Sector North, Block D, Apartment 1716, Fusion Building, Brasilia, Federal District, Brazil. Tel/Fax: +61-30517400 R-1716, E-mail: \\ gilpsilva2006@gmail.com
}

Received 2017 August 15; Accepted 2017 November 05

\begin{abstract}
Objectives: We proposed to investigate the possible effect and association of systemic inflammation (SI) and seminal parameter indicators in chronic hemodialysis patients.

Methods: This was a cross-sectional study. All the participants were subjected to a spermiogram with calculation of fertility index (FI), serum C-reactive protein (CRP) level, seminal transferrin(ST) level, as well as evaluation of the hormonal profile (HP). The sample consisting of 60 men (cases) undergoing hemodialysis for more than 6 months was subdivided into 3 groups: group 1 ( $n=30$, with inflammation, $C R P>5 \mathrm{mg} / \mathrm{L})$, group $2(n=30$, without inflammation, $C R P \leq 5 \mathrm{mg} / \mathrm{L})$, and group $3(n=30$, healthy men, $C R P \leq 1)$. Results: Age was similar in the 3 groups $(\mathrm{P}=0.43)$. FI, testosterone total and ST levels were significantly lower in the case groups than in the control group $(\mathrm{P}<0.001)$. Follicle stimulating hormone, luteinizing hormone, and prolactin levels were significantly higher in the case groups than in the control group $(\mathrm{P}<0.001)$. Between the subgroups of cases (groups 1 and 2 ), the inflammatory factor alone does not seem to interfere with the FI, HP, and ST level (P> 0.05). However, it significantly interfered with the FI, HP, and ST level when compared between the case groups and the control group $(\mathrm{P}<0.001)$. No correlation was observed between SI and analyzed parameters $(\mathrm{P}>0.05)$.

Conclusions: The results suggest that the SI alone has no effect and is not associated with the FI or ST level in a patient undergoing chronic hemodialysis.
\end{abstract}

Keywords: Chronic Kidney Disease, Hemodialysis, Seminal Parameter, Male Infertility, Seminal Transferrin and Systemic Chronic Inflammation, Fertility Index, Seminal Quality

\section{Background}

Systemic chronic inflammation (SCI) is a basic feature of chronic kidney disease (CKD)/end-stage renal disease (ESRD), especially in those undergoing hemodialysis (HD), and is related to genetics, uremia, dialysis, oxidative stress, and inflammatory factors (1). SCI is the result of the increased serum levels of proinflammatory cytokines such as interleukin (IL)- 6 and tumor necrosis factor (TNF- $\alpha$ ) as well as acute phase proteins such as C-reactive protein (CRP) and fibrinogen (1). It has now been well established that CKD/ESRD is a type of SCI (2).

Patients with CKD, especially HD, frequently present with subfertility/infertility, are characterized by poor seminal quality (3). The etiology of subfertility/infertility in these patients and in the general population is multifactorial, involving genetic, hormonal, immunological, oxidative, and inflammatory factors (4).
Subfertility/infertility in the male population is a clinical condition that affects approximately $15 \%$ of couples of childbearing age, with $50 \%$ of the masculine factor being manifested by changes either in sperm quality (concentration, motility, morphology, and sperm vitality) or in seminal plasma (5).

It has been reported that infection/inflammation of the genital tract increased cytokine level in seminal plasma and low quality of seminal parameters leads to subfertility/infertility (6).

However, the effect of SCI on the genital tract is poorly studied, especially in patients with CKD undergoing HD; around $40 \%-60 \%$ of whom suffer from SCI (1). We decided to investigate the possible effect and association of SCI using 2 seminal parameter indicators: fertility index (FI) and seminal transferrin (ST), in HD patients. 


\section{Methods}

\subsection{Recruitment, Inclusion and Exclusion}

The prospective study of prevalence was realized in the hemodialysis sector of the University hospital of the University of Brasilia, between July 2016 and December 2016, after approval by the research ethics committee of the faculty of health sciences of the University of Brasilia under number 53172316.9.0000.0030. The inclusion criteria were: age between 18 to 60 years, has been in HD for more than 6 months (cases), and absence of acute or chronic liver disease. The exclusion criteria included the presence of hemochromatosis or diseases of iron metabolism. Patients with hypogonadism and clinical conditions that could alter ST levels such as recent history of genitourinary tract infection, clinical signs of acute or chronic infection/inflammation, positive serology for hepatitis B, C, and human immunodeficiency virus (HIV), vascular access infection, leukocytosis, fever, as well as hypoproteinemia were not included in the study. All the participants were subjected to a spermiogram with calculation of FI, serum CRP level, and ST level, as well as an evaluation of the hormonal profile (HP) (follicle stimulating hormone-FSH; luteinizing hormone - $\mathrm{LH}$, total testosterone - TT and prolactin - PRL. The sample consisting of 60 men (cases) in high flow HD by vascular fistula access, $3 x$ week with duration of 4 hours / HD session, was subdivided into group 1 ( $\mathrm{n}=30$, with inflammation, CRP $>5 \mathrm{mg} / \mathrm{L}$ ) and group 2 ( $\mathrm{n}=30$, without inflammation, $\mathrm{CRP} \leq 5 \mathrm{mg} / \mathrm{L}$ ) as it is suggested by clinical practice guidelines and clinical practice recommendations for anemia in chronic kidney disease (7) as well as 30 healthy men (control) with lower cardiovascular risk (8) (CRP $\leq 1 \mathrm{mg} / \mathrm{L})$ from the health promotion outpatient clinic of the same hospital with renal function (glomerular filtration rate $>$ than $90 \mathrm{~mL} / \mathrm{min}$ per $1.73 \mathrm{~m}^{2}$ ), sperm without changes.

\subsection{Routine Collection of Blood and Semen}

The blood sample for analysis was collected from the arteriovenous fistula immediately before the first weekly hemodialysis session in the case group and on a previously scheduled day for the control group, always between 8:00 and 10:00 a.m. in the clinical laboratory of the same hospital to assay ST, FSH, LH, TT, and PRL. On the same day of blood collection, the semen was collected by voluntary masturbation in an appropriate environment at ambient temperature to perform spermiogram by manual method according to the guidelines of the world health organization (WHO) laboratory manual for the examination and processing of human semen 5 th ed (9). The seminal plasma preparation was centrifuging at $3500 \times \mathrm{g}$ for 20 minutes after 30 minutes liquefaction. The supernatant was collected into a new tube and held at $-20^{\circ} \mathrm{C}$ for the measurement of ST levels. ST and hormones belonging to the hormonal profile were measured by enzyme immunochemiluminescence using the Immulite 2000 / Siemens automatic analyzer. Specific kits were used for quantification, as well as calibrators and controls recommended by the manufacturer.

\subsection{Fertility Index (FI)}

IF was calculated according to Harvey (10) as follows: $\mathrm{FI}=$ sperm concentration $\left(\times 10^{6} / \mathrm{mL}\right) \times$ sperm motility $\times$ percentage of spermatozoa with normal morphology.

\subsection{Statistical Analysis}

After the normal distribution curve of the sample was verified by normality tests (Shapiro-Wilk), the one-way ANOVA test followed by the Bonferroni test was used for differences between 3 independent quantitative variables. To verify correlation between 2 independent quantitative variables Pearson correlation analysis was. Statistical significance was set at $\mathrm{P}<0.05$ to reject the null hypothesis. SPSS $^{\circledR}$ for Windows, version 20.0 was used.

\section{Results}

The age was similar in the 3 groups $(49.83 \pm 5.65 ; 49.10$ \pm 5.54 and $47.90 \pm 6.22, \mathrm{P}=0.43)$. FI, TT, and ST factors were significantly lower in the case groups than in the control group. Although there is no clinical hypogonadism (testosterone level was within normal range) in the sample population, FSH, LH, and PRL, levels were significantly higher in the cases than in the controls $(\mathrm{P}<0.001)$ (Table 1). The inflammatory factor, analyzed alone, did not seem to interfere with the FI, HP, and ST level between the subgroups of cases (groups 1 and 2, P > 0.05). However, it significantly interfered with the FI, HP, and ST level between the case groups and the control group (Table $1, \mathrm{P}<0.001$ ). No correlation was observed between the analyzed parameters and SCI (Table 2, P > 0.05).

\section{Discussion}

To our knowledge, the present study is the first to investigate the effect of SCI on 2 indicators of seminal parameters: ST and FI, in HD patients. These indicators can be useful in the initial evaluation of semen quality in patients with suspected subfertility/infertility, considering the ease and low cost at which sperm quality and seminal transferrin level can be tested.

The similar age of the groups $(P=0.430$, Table 1$)$ and the eugonadism of the sample population studied reduce 
Table 1. Comparative evaluation of age, fertility index, hormone profile and transferrin seminal level among groups $(x \pm S D),(N=30)$

\begin{tabular}{|c|c|c|c|c|c|c|c|}
\hline \multirow[t]{2}{*}{ Observed Parameters } & \multirow[t]{2}{*}{ Group 1} & \multirow[t]{2}{*}{ Group 2} & \multirow[t]{2}{*}{ Group 3} & \multirow[t]{2}{*}{ pa $^{\text {a Valors }}$} & \multicolumn{3}{|c|}{ Bonferroni' Test } \\
\hline & & & & & Group 1 and Group 2 & Group 1 and Group 3 & Group 2 and Group 3 \\
\hline Age, $y$ & $49.83 \pm 5.65$ & $49.10 \pm 5.54$ & $47.90 \pm 6.22$ & 0.430 & & & \\
\hline Fertility index ${ }^{b}$ & $0.66(0.33)$ & $1.05(1.3)$ & $5.54(1.3)$ & 00.000 & 00.29 & 00.000 & 00.000 \\
\hline FSH, $\mathrm{mIU} / \mathrm{mL}$ & $06.4 \pm 01.39$ & $06.18 \pm 01.00$ & $03.40 \pm 00.48$ & 00.000 & 01.00 & 00.000 & 00.000 \\
\hline $\mathrm{LH}, \mathrm{mIU} / \mathrm{mL}$ & $06.01 \pm 01.67$ & $15.81 \pm 02.61$ & $02.84 \pm 00.54$ & 00.000 & 01.00 & 00.000 & 00.000 \\
\hline Testosterone, ng/dL & $399.73 \pm 48.99$ & $422.33 \pm 66.24$ & $510.60 \pm 92.56$ & 00.000 & 01.00 & 00.000 & 00.000 \\
\hline Prolactin, ng/mL & $16.24 \pm 2.98$ & $16.52 \pm 02.91$ & $05.86 \pm 01.93$ & 00.000 & 01.00 & 00.000 & 00.000 \\
\hline Transferrin seminal, ng/mL & $37.90 \pm 06.22$ & $42.35 \pm 09.46$ & $73.32 \pm 06.81$ & 00.000 & 00.07 & 0.000 & 0.000 \\
\hline
\end{tabular}

Abbreviations: FSH, Follicle-Stimulating Hormone; LH, Luteinizing Hormone.

a One-way anova test.

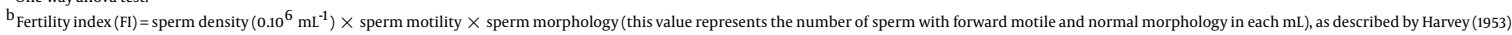

Table 2. Correlational Evaluation Pearson of C-Reactive Protein with Seminal Transferrin and Fertility Index in Group Case

\begin{tabular}{ccc}
\hline Observed Parameters & \multicolumn{2}{c}{ Group Case $(\mathbf{N}=\mathbf{6 0})$} \\
\hline C-reactive protein & $\mathrm{r}$ & P Value \\
\hline Seminal transferrin & & \\
\hline Fertility index & -0.166 & 0.204 \\
\hline
\end{tabular}

bias and confer reliability to the results. The sex hormones are important for seminal quality, as adequate spermatogenesis and seminal transferrin synthesis in the testicular gland, both of which are associated with seminal quality, require these hormones (11).

The HP identified in HD patients is often characterized by elevated serum levels of FSH, LH, and PRL, as well as low testosterone (hypogonadism) in approximately 1/3 of HD patients caused by blockages at one or more sites along the hypothalamic-pituitary-testicular (HPT) axis $(12,13)$.

Testicular level is due to dysfunction of Leydig cells because of pro-inflammatory cytokines, such as TNF- $\alpha$, IL-1, and interleukin 6(IL-6), which inhibit testicular Leydig cell steroidogenesis at the level of gene expression of different steroidogenic enzymes induced by CKD and HD (14), LH reduced clearance, as well as hyperprolactinemia caused by reduced clearance of PRL (12).

The sexual hormones (Table 1) in the case group in our study partially followed the pattern described above this is: high levels of FSH and LH, but TT levels within the limits of normality (eugonadics). Absence of clinical hypogonadism, in thesis, withdraw the hormonal factor in the pathophysiology of the changes found in the ST level and seminal parameter.

The FI and ST level are indicators frequently used in studies on patients suspected of suffering from subfertil- ity/infertility caused by seminal parameter change (15).

The FIs were significantly lower in the case subgroups than in the control group: 0.66(0.33) (group1) and 1.05 (1.3) (group 2) vs. 5.54 (1.3) (group 3), $\mathrm{P}<0.001$. Xu et al. (16) found similar results in a uremic population: 0.68 (2.08) for case and 7.7 (13.51) for control.

ST levels were significantly lower in the case subgroups than in the control group $37.90 \pm 06.22 \mathrm{ng} / \mathrm{mL}$ (group 1) and $42.35 \pm 09.46 \mathrm{ng} / \mathrm{mL}$ (group 2) vs. $73.32 \pm 06.81$ $\mathrm{ng} / \mathrm{mL}$ (group 3), $\mathrm{P}<0.001$. This finding has been corroborated by a study by Bharshankar and Bharshankar (17), who found that mean seminal plasma transferrin concentration in fertile men was $5.35 \pm 2.07 \mathrm{mg} / \mathrm{dL}$ and that in normozoospermic subject was $4.63 \pm 2.50 \mathrm{mg} / \mathrm{dL}$, which was significantly higher $(\mathrm{P}<0.001)$ than that in oligozoospermic, azoospermic, and post-vasectomized subjects. The reasons for which ST levels are lower in patients with poor seminal quality are unknown, however, it is hypothesized that it is because IL- 6 reduces transferrin secretion by the Sertoli cells of the testes (18).

The reduction in seminal quality found in patients undergoing HD and reflected in the FI and ST levels analyzed in this study is multifactorial (prevalence of uremia and hormonal, immunological, oxidative, and inflammatory factors) (19).

It is an established fact that inflammation/infection of the male urogenital tract reduces seminal quality due to an increase in the levels of seminal cytokines (18).

The effect of chronic systemic inflammation as a modifying factor of seminal parameter is little studied. After the contribution of uremic factor in the lowering of seminal quality in patients with renal failure was recognized $(16,20)$, the contribution of the oxidative/inflammatory factors was emphasized $(21,22)$. Patients undergoing hemodialysis can be characterized by increased levels of oxidative stress and inflammation (23). The relation of inflammation and oxidative stress to overproduction of reac- 
tive oxygen species (ROS) in HD is well known (24). This relation is attributed to the ability of ROS to activate nuclear factors such as nuclear factor kappa B (NF- $\kappa \mathrm{B})$, which is an inducer of the synthesis of pro-inflammatory cytokines such as IL-6 and TNF- $\alpha$, and the consequent elevation of acute phase proteins such as fibrinogen and CRP (24). In addition to being an inflammation inducer (24), ROS have an important direct contribution to the seminal parameter changes, promoting substantial adverse effects on the structural and functional integrity of sperms by protein, glycogen, lipid, and DNA peroxidation (25). They can therefore be considered partially responsible for defective morphology and function of the sperms of male patients with subfertility/infertility (25).

As SCI is almost always present in patients undergoing $\mathrm{HD}(1)$, it is plausible to postulate that hypercytokinemia induced in such patients can profoundly affect vascular testicular permeability and can reach the interstitial compartment of the testis (26). Hypercytokinemia, which is a part chronic inflammation in HD patients, modulates interactions among immunological, oxidative, and inflammatory mediators, which are responsible for sperm dysfunction (18). This would cause profound and direct changes in the physiology of the hematotesticular barrier, stimulate the testicular macrophages to produce different pro and anti-inflammatory cytokines, destroy the local paracrine/autocrine systems, as well as other mechanisms, which are responsible for maintaining the immune privileged condition of the testes $(26,27)$.

Thus, we cannot affirm that neither the significant differences (FI, PH, and ST) found between the case groups and the control group $(\mathrm{P}<0.001)$, nor the absence of effect of the inflammatory factor on $\mathrm{FI}, \mathrm{PH}$, and ST in the subgroups of cases (groups 1 and 2) $(\mathrm{P}>0.05)$ were due solely to the inflammatory factor analyzed. The absence of correlation of the inflammatory factor with IF and ST (Table 2) may reinforce the multifactorial etiology of the seminal alterations found among the groups. This study has 2 limitations: the lack of measurement of total seminal antioxidant capacity and a small sample size. The results suggest that the inflammatory factor alone has no effect and is not associated with the fertility index or seminal transferrin level in a patient undergoing chronic hemodialysis.

\section{Footnotes}

Authors' Contribution: Gilmar Pereira Silva and Fabiana Pirani Carneiro, equal contributors. Gilmar Pereira Silva drafted the manuscript. Fabiana Pirani Carneiro and Vitor Pereira Xavier Grangeiro critically reviewed it and make addition. All authors declared the final version of manuscript.
Competing Interests: The authors declare that they have no competing interests.

Ethical Approval: Approved by the research ethics committee of the faculty of health Sciences of the University of Brasilia under number 53172316.9.0000.0030.

Informed Consent: Informed consent was obtained from all individual participants included in the study.

\section{References}

1. Akchurin OM, Kaskel F. Update on inflammation in chronic kidney disease. Blood Purif. 2015;39(1-3):84-92. doi: 10.1159/000368940. [PubMed: 25662331].

2. Yilmaz MI, Carrero JJ, Axelsson J, Lindholm B, Stenvinkel P. Low-grade inflammation in chronic kidney disease patients before the start of renal replacement therapy: sources and consequences. Clin Nephrol. 2007;68(1):1-9. doi: 10.5414/CNP68001. [PubMed: 17703829].

3. Palmer BF, Clegg DJ. Gonadal dysfunction in chronic kidney disease. Rev Endocr Metab Disord. 2017;18(1):117-30. doi: 10.1007/s11154-016-93859. [PubMed: 27586847].

4. Ventimiglia E, Montorsi F, Salonia A. Comorbidities and male infertility: a worrisome picture. Curr Opin Urol. 2016;26(2):146-51. doi: 10.1097/MOU.0000000000000259. [PubMed: 26765042].

5. Coutton C, Fissore RA, Palermo GD, Stouffs K, Toure A. Male Infertility: Genetics, Mechanism, and Therapies. Biomed Res Int. 2016;2016:7372362. doi: 10.1155/2016/7372362. [PubMed: 26942199].

6. Azenabor A, Ekun AO, Akinloye O. Impact of Inflammation on Male Reproductive Tract. J Reprod Infertil. 2015;16(3):123-9. [PubMed: 26913230].

7. National Kidney F.Kdoqi. KDOQI Clinical Practice Guidelines and Clinical Practice Recommendations for Anemia in Chronic Kidney Disease. Am J Kidney Dis. 2006;47(5 Suppl 3):S11-145. doi: 10.1053/j.ajkd.2006.03.010. [PubMed:16678659].

8. Willerson JT, Ridker PM. Inflammation as a cardiovascular risk factor. Circulation. 2004;109(21 Suppl 1):II2-10. doi: 10.1161/01.CIR.0000129535.04194.38. [PubMed: 15173056].

9. World Health Organization. WHO laboratory manual for the examination and processing of human semen. 2010.

10. Harvey C. A fertility index derived from semen analysis. J Clin Pathol. 1953;6(3):232-6. doi:10.1136/jcp.6.3.232. [PubMed: 13084771].

11. Ramaswamy S, Weinbauer GF. Endocrine control of spermatogenesis: Role of FSH and LH/ testosterone. Spermatogenesis. 2014;4(2). e996025. doi: 10.1080/21565562.2014.996025. [PubMed: 26413400].

12. Lo JC, Beck GJ, Kaysen GA, Chan CT, Kliger AS, Rocco MV, et al. Hyperprolactinemia in end-stage renal disease and effects of frequent hemodialysis. Hemodial Int. 2017;21(2):190-6. doi: 10.1111/hdi.12489. [PubMed: 27774730].

13. Grossmann M, Hoermann R, Ng Tang Fui M, Zajac JD, Ierino FL, Roberts MA. Sex steroids levels in chronic kidney disease and kidney transplant recipients: associations with disease severity and prediction of mortality. Clin Endocrinol (Oxf). 2015;82(5):767-75. doi: 10.1111/cen.12656. [PubMed: 25378236].

14. Tremblay JJ. Molecular regulation of steroidogenesis in endocrine Leydig cells. Steroids. 2015;103:3-10. doi: 10.1016/j.steroids.2015.08.001. [PubMed: 26254606].

15. Boe-Hansen GB, Rego JP, Crisp JM, Moura AA, Nouwens AS, Li $\mathrm{Y}$, et al. Seminal plasma proteins and their relationship with percentage of morphologically normal sperm in 2-year-old Brahman (Bos indicus) bulls. Anim Reprod Sci. 2015;162:20-30. doi: 10.1016/j.anireprosci.2015.09.003. [PubMed: 26417650]. 
16. Xu L, Xu H, Zhu X, Zhang J, Ma M, Shi X. Effect of uremia on semen quality and reproductive function in humans. Cell Biochem Biophys. 2012;62(1):29-33. doi:10.1007/s12013-011-9254-9. [PubMed: 21826526].

17. Bharshankar RN, Bharshankar JR. Relationship of seminal plasma transferrin with seminal parameters in male infertility. Indian J Physiol Pharmacol. 2000;44(4):456-60. [PubMed: 11214501].

18. Fraczek M, Kurpisz M. Cytokines in the male reproductive tract and their role in infertility disorders. J Reprod Immunol. 2015;108:98-104 doi: 10.1016/j.jri.2015.02.001. [PubMed: 25796532].

19. Dohle G. ClinicalUro-Andrology. Springer; 2015. Male factors in couple's infertility; p. 197-201.

20. Lehtihet M, Hylander B. Semen quality in men with chronic kidney disease and its correlation with chronic kidney disease stages. Andrologia. 2015;47(10):1103-8. doi: 10.1111/and.12388. [PubMed: 25487067].

21. Cachofeiro V, Goicochea M, de Vinuesa SG, Oubina P, Lahera V, Luno J. Oxidative stress and inflammation, a link between chronic kidney disease and cardiovascular disease. Kidney Int Suppl. 2008;(111):S4-9. doi:10.1038/ki.2008.516. [PubMed: 19034325].

22. Aitken RJ. Oxidative stress and the etiology of male infertility. J Assist Reprod Genet. 2016;33(12):1691-2. doi: 10.1007/s10815-016-0791-4
[PubMed: 27544275].

23. Hagmann H, Brinkkoetter PT. ROS and oxidative stress in CKD patients: is it the mitochondria that keeps CKD patients in bed?. Nephrol Dial Transplant. 2015;30(6):867-8. doi: 10.1093/ndt/gfv052. [PubMed: 25735768].

24. Tucker PS, Scanlan AT, Dalbo VJ. Chronic kidney disease influences multiple systems: describing the relationship between oxidative stress, inflammation, kidney damage, and concomitant disease. Oxid Med Cell Longev. 2015;2015:806358. doi: 10.1155/2015/806358. [PubMed: 25861414].

25. Asadi N, Bahmani M, Kheradmand A, Rafieian-Kopaei M. The Impact of Oxidative Stress on Testicular Function and the Role of Antioxidants in Improving it: A Review. J Clin Diagn Res. 2017;11(5):IE01-5. doi: 10.7860/JCDR/2017/23927.9886. [PubMed: 28658802].

26. Zhang H, Yin Y, Wang G, Liu Z, Liu L, Sun F. Interleukin-6 disrupts blood-testis barrier through inhibiting protein degradation or activating phosphorylated ERK in Sertoli cells. Sci Rep. 2014;4:4260. doi: 10.1038/srep04260. [PubMed: 24584780].

27. Chen H, Mruk D, Xiao X, Cheng CY. Male Hypogonadism. Springer; 2017. Human Spermatogenesis and Its Regulation; p. 49-72. doi: 10.1007/978-3-319-53298-1_3. 